\title{
LEBESGUE THEORY ON A BOOLEAN ALGEBRA
}

BY

JOHN M. H. OLMSTED

\section{INTRODUCTION AND DEFINITIONS}

Associated with a real-valued point function everywhere finite is the setvalued function

$$
E(\alpha)=\underset{x}{E}[f(x)>\alpha]
$$

The function $E(\alpha)$ in turn determines $f(x)$ :

$$
f(x)=\sup \underset{\alpha}{E}[x \in E(\alpha)] .
$$

A necessary and sufficient set of conditions that a function, $E(\alpha)$, from real numbers to subsets of a set $S$, be associated, as above, with a real-valued point function everywhere finite is

1. $E(\alpha) \downarrow$ as $\alpha \uparrow ;$

2. $\sum_{n=1}^{\infty} E(-n)=S, \prod_{n=1}^{\infty} E(n)=0$;

3. $\sum_{n=1}^{\infty} E(\alpha+1 / n)=E(\alpha)$ for every $\alpha$.

It is possible, therefore, to consider $f(x)$ and $E(\alpha)$ as different aspects of the same function, one more natural for algebraic combinations of functions, and the other receiving emphasis in a theory of integration. However, one may restrict oneself entirely to the second aspect. For example, if

$$
\begin{aligned}
& A(\alpha)=\underset{x}{E}[f(x)>\alpha], \\
& B(\alpha)=\underset{x}{E}[g(x)>\alpha], \\
& C(\alpha)=\underset{x}{E}[f(x)+g(x)>\alpha],
\end{aligned}
$$

then

$$
C(\alpha)=\sum_{\beta} \Pi[A(\beta), B(\alpha-\beta)]
$$

where the summation is formed with respect to any dense set of real numbers $\beta$.

This fact frees us altogether from the point aspect of functions and enables us to extend our attention to functions whose values are elements of a Boolean algebra and which cannot in general be regarded as point functions. If there is a measure function we may have "almost everywhere" instead of

Presented to the Society, September 11, 1940; received by the editors December 9, 1940. 
"everywhere" finite, and consider the new Boolean algebra of equivalence classes of elements differing by elements of measure zero.

Chapters one and two deal with algebraic manipulations of functions and extensions of partially ordered linear spaces with element 1 as defined by Freudenthal $[1]\left({ }^{1}\right)$. It is proved that any such space can be extended to a partially ordered space of the same type which is also a ring, the multiplicative unit of which is the element 1 . Spaces analogous to the space $M$ of bounded measurable functions and, in the case of a measure on the Boolean algebra, to the Lebesgue $L^{p}$ spaces are treated in Chapters three and four. In Chapter five the notions of absolute continuity, indefinite integral, and derivative are studied. It is shown that in terms of these concepts the Nikodym theorem (cf. Saks [2, chap. 1, §14]) concerning absolutely continuous functions is again available. Chapter six contains remarks on conjugate spaces, regularity for a partially ordered linear space as defined by Kantorovitch [3], and spaces of the type defined by Orlicz [4].

Spaces of functions based on a Boolean algebra have been considered from a different approach by Carathéodory [5]. The present paper rests particularly on the work of Kantorovitch [3] and Freudenthal [1].

Terminology will be in large part that used by Birkhoff in his Lattice Theory [8]. Definitions given there will not be repeated here. The symbols $\checkmark$ and $\wedge$ will be used to represent the "sup" and "inf," respectively, of a collection of elements of a lattice. The notation of Kantorovitch, $a^{-}=\bigvee(-a, 0)$, where $a$ is an element of a vector lattice, will be retained. A vector lattice will also be called a $K$-space (Kantorovitch space). If a vector lattice has an element 1 , that is, an element $1>0$ with the property that

$$
\wedge(a, 1)=0 \rightarrow a=0,
$$

it will also be called an $F$-space (Freudenthal space). Real numbers will in general be denoted by Greek letters, integers generally by $m, n$. The letters, $p, q$ will also on occasion be used for real numbers, generally $\geqq 1$. Latin letters will generally be reserved for elements of a lattice. We shall frequently write $f(\xi)$ to mean the function $f(\xi)$ itself rather than its value at a point $\xi$. It will be also convenient to denote the function by the single letter $f$ if there is no danger of confusion.

Throughout this paper the following fact holds. If the Boolean algebra under consideration is an algebra of point sets (cf. Stone [6], Wecken [7]), the theory reduces to the classical case.

\section{THE SPACE $\Omega$}

Notation. Let $B$ be a $\sigma$-complete Boolean algebra. Denote by $\Omega$ the set of all functions, $f(\boldsymbol{\alpha})$, from real numbers to elements of $B$, satisfying the conditions:

(1) Numbers in brackets indicate references at the end of the paper. 
1. $f(\alpha) \downarrow$ as $\alpha \uparrow$,

2a. $\vee_{\alpha} f(\alpha)=1$,

2b. $\wedge_{\alpha} f(\alpha)=0$,

3. $\bigvee_{\beta>\alpha} f(\beta)=f(\alpha)$ for every $\alpha$.

Remark. Because of the monotoneity condition 1 , all $\vee$ and $\wedge$ indicated exist.

With proper definitions of ordering, addition, and multiplication by real numbers, the set $\Omega$ can be regarded as a $\sigma$-complete $F$-space. The symbol $\Omega$ will be used to designate this space as well as the set of its elements. We shall now define and discuss the operations in the space $\Omega$.

1. Ordering. We state the following definition.

Definition. If $f(\xi)$ and $g(\xi)$ are elements of $\Omega$, we define $f(\xi) \leqq g(\xi)$ to mean that $f(\xi) \leqq g(\xi)$ for every $\xi, f(\xi)=g(\xi)$ to mean that $f(\xi)=g(\xi)$ for every $\xi$, and $f(\xi)<g(\xi)$ to mean that $f(\xi) \leqq g(\xi)$, but $f(\xi) \neq g(\xi)$.

Remark. Under this definition, $\Omega$ becomes a partially ordered set.

TheOREM 1.1. If $f_{n}, g \in \Omega, f_{n} \leqq g$, then $f=\bigvee_{n} f_{n}$ exists and

$$
f(\xi)=\bigvee_{n} f_{n}(\xi)
$$

If $f_{n}, g \in \Omega, f_{n} \geqq g$, then $f=\bigwedge_{n} f_{n}$ exists and

$$
f(\xi)=\bigvee_{m=1}^{\infty} \wedge f_{n}(\xi+1 / m) .
$$

If $f_{1}, \cdots, f_{n} \in \Omega$, then $h=\bigvee\left(f_{1}, \cdots, f_{n}\right)$ and $f=\wedge\left(f_{1}, \cdots, f_{n}\right)$ exist and

$$
f(\xi)=\wedge\left(f_{1}(\xi), \cdots, f_{n}(\xi)\right) .
$$

Proof is omitted.

2. Addition. We state the following definition.

Definition. If $f(\xi)$ and $g(\xi)$ are elements of $\Omega$, we define their sum, $h(\xi)=f(\xi)+g(\xi)$ to be the function

$$
h(\xi)=\bigvee_{\beta} \wedge[f(\beta), g(\xi-\beta)],
$$

where the "sup" is formed with respect to any countable dense set of real numbers $\beta$.

To speak of addition as thus defined we must show that the values of $h(\xi)$ are independent of the dense set chosen and that the function so defined belongs to $\Omega$. Let us first observe that due to the normalization condition 3 a function in $\Omega$ is completely determined if its values over a dense set of real numbers are known. We state two lemmas: 
Lemma 1. If $a_{n} \uparrow$ and $b_{n} \uparrow$ are two increasing sequences of elements of $B$, then

Proof is omitted.

$$
\left.\underset{n}{\bigvee} \bigwedge=\left(a_{n}, b_{n}\right)=\wedge \underset{m}{\bigvee} a_{m}, \underset{n}{\bigvee} b_{n}\right)
$$

LeMma 2. If $h=f+g$, as defined above, then for any $\xi$ the value $h(\xi)$ is independent of the choice of the dense set $\beta$.

To verify this it will be sufficient to show that for any $\rho$

$$
\bigvee_{\beta} \wedge[f(\beta), g(\xi-\beta)] \geqq \wedge[f(\rho), g(\xi-\rho)] .
$$

The left-hand side is greater than or equal to

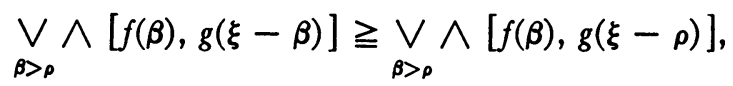

which by the distributive law for $B$ is equal to

$$
\wedge\left[\bigvee_{\beta>\rho} f(\beta), g(\xi-\rho)\right]=\wedge[f(\rho), g(\xi-\rho)]
$$

Theorem 1.2. If $f$ and $g \in \Omega$, then $h=f+g \in \Omega$.

Proof. We shall verify the second of the three conditions.

Ad 2a: Form the sum with respect to a dense set $\{\rho\}$. Then

$$
\begin{aligned}
\underset{n}{\vee} \wedge \wedge[f(\rho), g(-n-\rho)] & =\underset{\rho}{\vee} \vee \wedge \wedge[f(\rho), g(-n-\rho)] \\
& =\bigvee_{\rho}\left[\wedge\left[f(\rho), \bigvee_{n} g(-n-\rho)\right]\right] \\
& =\bigvee_{\rho}[\wedge[f(\rho), 1]]=1 .
\end{aligned}
$$

Ad 2b: We have

$\wedge \vee \wedge[f(\rho), g(n-\rho)]$

$$
\begin{aligned}
& =\bigwedge_{n} \vee\left[\bigvee_{\rho>n} \wedge[f(\rho), g(2 n-\rho)], \underset{\rho<n}{\vee} \wedge[f(\rho), g(2 n-\rho)]\right] \\
& \leqq \wedge \wedge_{n} \vee\left[\bigvee_{\rho>n} \wedge[f(n), 1], \underset{\rho<n}{\vee} \wedge[1, g(n)]\right]=\bigwedge_{n} \vee[f(n), g(n)] .
\end{aligned}
$$

Because of the monotoneity of $f(\xi)$ and $g(\xi)$ we can apply Lemma 1 . The last expression above is therefore equal to zero.

TheOREM 1.3. Addition is commutative.

Proof is omitted.

Theorem 1.4. Addition is associative.

Proof is based on the associative and distributive laws for $B$. 
3. Multiplication by real numbers. We give first a definition.

Definition. If $\alpha>0, g=\alpha f$ is the function

$$
g(\xi)=f(\xi / \alpha) .
$$

The function $g=-f$ is given by

$$
g(\xi)=1-\bigwedge_{\rho<-\xi} f(\rho)=1-\bigwedge_{n=1}^{\infty} f(-\xi-1 / n) .
$$

The function $g=0=0 f$ is given by

$$
g(\xi)=0(\xi)=\left\{\begin{array}{l}
1, \xi<0 \\
0,0 \leqq \xi
\end{array}\right.
$$

If $\alpha<0, g=\alpha$ is the function $-[(-\alpha) f]$.

There is no difficulty in showing in each case that the defined function belongs to $\Omega$.

TheORem 1.5. 1. $\alpha(\beta f)=(\alpha \beta) f, 2 .(\alpha+\beta) f=\alpha f+\beta f, 3 . \alpha(f+g)=\alpha f+\alpha g$, for all real numbers, $\alpha, \beta$, and for any elements $f, g$ of $\Omega$.

Proof. Ad 1: The following statements, from which the general form follows, are easily proved:

(1) $-(,-f)=f$,

(2) $-(\alpha f)=\alpha(-f)$, if $\alpha>0$,

(3) $1 \cdot f=f$,

(4) $(-1) f=-f$,

(5) $-(0)=0$, where $0=0(\xi)$,

(6) $\alpha(0(\xi))=0(\xi)$ for every $\alpha$,

(7) $\alpha(\beta f)=(\alpha \beta) f$, if $\alpha=0$, or if $\beta=0$,

(8) $\alpha(\beta f)=(\alpha \beta) f$, if $\alpha>0$, and $\beta>0$.

Ad 2: Proof involves such preliminaries as showing that $f+0=f$ and that $f+(-f)=0$, for any $f$. The only one involving any difficulty is the proof that $f+(-f)=0$. For any real number $\xi$

$$
[f+(-f)](\xi)=\vee_{\rho} \wedge\left[1-\bigwedge_{\beta<-\rho} f(\beta), f(\xi-\rho)\right] .
$$

If $\xi \geqq 0$, there is no difficulty in showing that this expression is equal to zero. Now let us assume that $\xi<0$, and show that the above expression is equal to 1 . We know that it is greater than or equal to

$$
\vee_{\rho} \wedge[1-f(\xi / 2-\rho), f(\xi-\rho)]
$$

Setting $2 \alpha=-\xi, \alpha>0$, and letting $\rho$ run over the infinite range of values $n \alpha$, for all integers $n$ (we can add these values to the dense set of $\rho$ without alter- 
ing the supremum), we see that the expression above is greater than or equal to

$$
\begin{aligned}
& \vee \backslash \cdots \wedge[f(-n \alpha), 1-f(-(n-1) \alpha)], \cdots, \\
& \quad \begin{array}{l}
\wedge f(-\alpha), 1-f(0)], \wedge[f(0), 1-f(\alpha)], \cdots] \\
\quad=\bigvee_{n} \vee[\wedge[f(-n \alpha), 1-f(-(n-1) \alpha)], \cdots, \\
\quad \wedge[f((n-1) \alpha), 1-f(n \alpha)]]
\end{array}
\end{aligned}
$$

Iterating the relation $\vee[\wedge(a, 1-b), \wedge(b, 1-c)]=\wedge(a, 1-c)$, where $a \geqq b \geqq c$, we obtain the result that the last expression above is equal to

$$
\bigvee_{n} \wedge[f(-n \alpha), 1-f(n \alpha)]=\wedge\left[\bigvee_{n} f(-n \alpha), 1-\bigwedge_{n} f(n \alpha)\right]=\wedge(1,1)=1
$$

Ad 3: Proof is not difficult.

This completes the proof of Theorem 1.5.

It follows immediately from the definition of ordering that a necessary and sufficient condition that an element $f(\xi)$ of $\Omega$ be $\geqq 0$ is that $f(\xi)=1$ for $\xi<0$. It is now easily proved that if $f>0, g>0, \alpha>0$, then $f+g>0, \alpha f>0$. Furthermore, if $f \in \Omega$, then $f^{+} \in \Omega$. It is given by

$$
f^{+}(\xi)=\left\{\begin{array}{cc}
1, & \xi<0, \\
f(\xi), & \xi \geqq 0 .
\end{array}\right.
$$

Theorem 1.6. The space $\Omega$ is a $\sigma$-complete F-space.

Proof. We have only to show that there exists an element $1 \in \Omega$. Such an element is the function

$$
1(\xi)=\left\{\begin{array}{l}
1, \xi<1 \\
0, \xi \geqq 1
\end{array}\right.
$$

Corresponding to any element $a$ of $B$ we define the function $a(\xi)$ :

$$
a(\xi)=\left\{\begin{array}{l}
1, \quad \xi<0, \\
a, 0 \leqq \xi<1, \\
0,1 \leqq \xi .
\end{array}\right.
$$

If $a$ is the element $\mathbf{0}$ or 1 of the Boolean algebra, the corresponding function is the element 0 or 1 , previously defined, of the space $\Omega$. In case there is no danger of confusion, we shall use $a$ to denote the function itself. We shall borrow a term from real variable theory and call these functions "characteristic functions."

Definition. Two elements, $a$ and $b$, of a Boolean algebra are "disjoint" if $\wedge(a, b)=0$. Two elements $a$ and $b$, of $a$ vector lattice are "disjoint" if $\wedge(|a|,|b|)$ $=0$. 


\section{RING PROPERTY OF $\Omega$}

The space $\Omega$ bears a closer resemblance to a space of point functions than the general $\sigma$-complete $F$-space, for it admits the introduction of a multiplication and a raising of positive elements to powers, satisfying the usual laws.

Notation. We denote by $\Omega^{+}$the elements of $\Omega$ which are $\geqq 0$.

DEFINITION. If $f$ and $g$ are elements of $\Omega^{+}$, we define their product, $h=f g$, to be the function

$$
h(\xi)=\left\{\begin{array}{l}
1, \xi<0, \\
\bigvee_{\rho>0} \wedge[f(\rho), g(\xi / \rho)], \xi \geqq 0,
\end{array}\right.
$$

where the least upper bound is formed with respect to any countable set of points, $\rho$, dense in the set of positive real numbers.

As in the case of sums, the values of $h(\xi)$ are independent of the dense set chosen. Similarly we have

Theorem 1.7. If $f, g \in \Omega^{+}$, then $f g \in \Omega^{+}$.

Proof is omitted.

Let us observe that if $f \geqq 0, g \geqq 0$, then a necessary and sufficient condition that $f g=0$ is that $\wedge(f, g)=0$, that is,

$$
\wedge[f(0), g(0)]=0 .
$$

Definition. If $f \in \Omega^{+}$and if $p$ is a positive real number, then $g=f^{p}$ is defined:

$$
g(\xi)=\left\{\begin{array}{cc}
1, & \xi<0, \\
f\left(\xi^{1 / p}\right), & \xi \geqq 0 .
\end{array}\right.
$$

We now state the following theorem about functions $\in \Omega^{+}$:

THEOREM 1.8. 1. $f g=g f, 2$. $(f g) h=f(g h), 3 . f(g+h)=f g+f h, 4 . f 0=0$, 5. $f \cdot 1=f, 6$. if $\alpha>0,(\alpha f)(g)=\alpha(f g), 7 \cdot f^{p} f^{q}=f^{p+q}$, for $p>0, q>0,8 . f^{p} g^{p}=(f g)^{p}$, for $p>0$, 9. if $f g=0$, then $(f+g)^{p}=f^{p}+g^{p}, 10 . a^{p}=a$, for $p>0,11 . \vee\left[f^{p}, g^{p}\right]$ $=[\bigvee(f, g)]^{p}, \wedge\left[f^{p}, g^{p}\right]=[\wedge(f, g)]^{p}$, for $p>0,12 . \bigvee_{n}\left(f_{n}^{p}\right)=\left(\bigvee_{n} f_{n}\right)^{p}$, for $p>0$.

Proof is omitted.

Definition. For arbitrary elements of $\Omega$ we define

$$
f g=f^{+} g^{+}+f^{-} g^{-}-f^{+} g^{-}-f^{-} g^{+} .
$$

Lemma. $(f g)^{+}=f^{+} g^{+}+f^{-} g^{-},(f g)^{-}=f^{+} g^{-}+f^{-} g^{+}$.

Proof. The statement of the lemma is equivalent to stating

$$
\wedge\left(f^{+} g^{+}+f^{-} g^{-}, f^{+} g^{-}+f^{-} g^{+}\right)=0,
$$


which is equivalent to saying

$$
\left(f^{+} g^{+}+f^{-} g^{-}\right)\left(f^{+} g^{-}+f^{-} g^{+}\right)=0,
$$

and by the distributive, associative, and commutative laws this is true because $f^{+} f^{-}=g^{+} g^{-}=0$.

We are now in a position to state a theorem regarding multiplication of arbitrary elements of $\Omega$ :

THEOREM 1.9. 1. $f g=g f, 2 .(f g) h=f(g h), 3 . f(g+h)=f g+f h, 4 . f \cdot 0=0$, 5. $f \cdot 1=f, 6 .(\alpha f) g=\alpha(f g), 7 .|f g|=|f| \cdot|g|$, 8. $|\alpha f|=|\alpha| \cdot|f|$.

Proof is omitted.

Definition. If $f \geqq 0$, we define

$$
f_{a}(\xi)=\left\{\begin{array}{cc}
1, & \xi<0 \\
\wedge[f(\xi), a], & \xi \geqq 0 .
\end{array}\right.
$$

For arbitrary $f \in \Omega$, we define

$$
f_{a}=\left(f^{+}\right)_{a}-\left(f^{-}\right)_{a} .
$$

One could now prove such statements as the following:

$$
\left(f^{+}\right)_{a}=\left(f_{a}\right)^{+} ;(f+g)_{a}=f_{a}+g_{a} ;\left(f_{a}\right)_{b}=\left(f_{b}\right)_{a}=f_{\wedge(a, b)} ;(f g)_{a}=f_{a} g_{a} ;
$$

if $f \geqq 0, f_{a}=\bigvee_{n} \wedge(f, n a)$. Results of this nature will be used after the notion of integral has been introduced.

Definition. A function $f(\xi)$ is "bounded above" if there exists an $\alpha$ such that $f(\alpha)=0 ; f(\xi)$ is "bounded below" if there exists a $\beta$ such that $f(\beta)=1 ; f(\xi)$ is "bounded" if it is bounded above and bounded below.

Definition. A simple function is one which takes on only a finite number of values. A super-simple function is a simple function which is greater than or equal to zero and which takes on at most one value other than 0 and 1.

Theorem 1.10. Any positive simple function can be represented as a sum of disjoint super-simple functions. Any simple function can be represented as a linear combination of disjoint "characteristic functions."

Proof. We prove the first part. Let the given positive simple function be

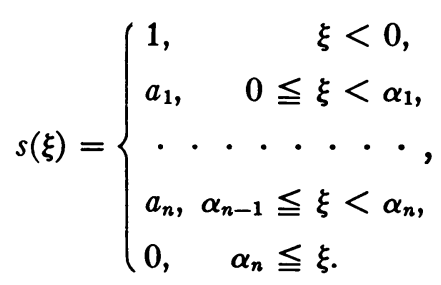


Define

$$
u(\xi)=\left\{\begin{array}{lrr}
1, & \xi<0, \\
a_{2}, & 0 \leqq \xi<\alpha_{2}, \\
\cdot . & \cdot \cdot \cdot, \\
a_{n}, & \alpha_{n-1} \leqq \xi<\alpha_{n}, \\
0, & \alpha_{n} \leqq \xi ;
\end{array} \quad t(\xi)=\left\{\begin{array}{lr}
1, & \xi<0, \\
a_{1}-a_{2}, & 0 \leqq \xi<\alpha_{1}, \\
0, & \alpha_{1} \leqq \xi ;
\end{array}\right.\right.
$$

and $v(\xi)=u(\xi)+t(\xi)$. Then

$$
v(\xi)=\vee\left\{\begin{array}{l}
t(\xi), \wedge\left[a_{2}, t\left(\xi-\alpha_{2}\right)\right], \cdots, \wedge\left[a_{n}, t\left(\xi-\alpha_{n}\right)\right], \\
u(\xi), \wedge\left[u\left(\xi-\alpha_{1}\right), a_{1}-a_{2}\right],
\end{array}\right.
$$

and by direct comparison $v(\xi)=s(\xi)$ for all $\xi$. Therefore a positive function with at most $n+2$ values can be represented as the sum of a positive function with at most $n+1$ values and a super-simple function, for any positive integer $n$. Induction completes the proof.

Given any two simple functions, $s$ and $t$, we may write them as linear combinations of the same characteristic functions, that is, in the form

$$
s=\alpha_{1} a_{1}+\cdots+\alpha_{n} a_{n}, \quad t=\beta_{1} a_{1}+\cdots+\beta_{n} a_{n} .
$$

Using the distributive laws, we can now write:

$$
\begin{aligned}
s+t & =\left(\alpha_{1}+\beta_{1}\right) a_{1}+\cdots+\left(\alpha_{n}+\beta_{n}\right) a_{n}, \\
s t & =\left(\alpha_{1} \beta_{1}\right) a_{1}+\cdots+\left(\alpha_{n} \beta_{n}\right) a_{n} .
\end{aligned}
$$

Theorem 1.11. The simple functions of $\Omega$ form an $F$-space possessing the ring property.

Proof is omitted.

Since $\Omega$ is a $\sigma$-complete vector lattice it admits the introduction of a limit (cf. Birkhoff [8, chap. 2]), with respect to which we can state:

THEOREM 1.12. The space of simple functions is dense, in the sense of this limit, in the space $\Omega$.

Proof. Suppose $f \geqq 0$. Take any countable dense set of positive real numbers, $\rho_{n}$, and letting $0<\beta_{1}<\cdots<\beta_{n}$ be the rearrangement of $\rho_{1}, \cdots, \rho_{n}$ according to magnitude, define

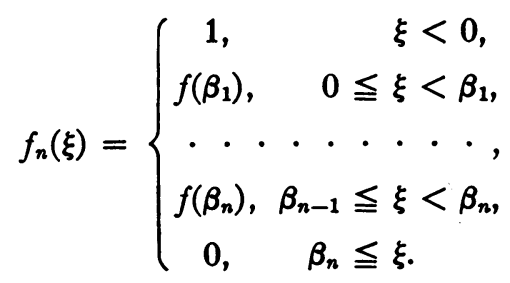


Then $f_{n}$ is an increasing sequence of simple functions and

$$
\bigvee_{n} f_{n}(\xi)=\bigvee_{\rho>\xi} f(\rho)=f(\xi) \text {. }
$$

Finally, if $f$ is an arbitrary function $\in \Omega$, write $f=f^{+}-f^{-}$, and let $\bigvee_{n} g_{n}=f^{+}$, $\bigvee_{n} h_{n}=f^{-}, f_{n}=g_{n}-h_{n}$. Then $f_{n}$ is a simple function, and

$$
\lim f_{n}=\lim g_{n}-\lim h_{n}=f^{+}-f^{-}=f .
$$

THEOREM 1.13. Let $f, g \in \Omega^{+}$, and let $s_{n}$ and $t_{n}$ be simple functions $\in \Omega^{+}$. If $s_{n} \uparrow f, t_{n} \uparrow g$, then $s_{n}+t_{n} \uparrow f+g$ and $s_{n} t_{n} \uparrow f g$.

Proof. The first statement is proved by Birkhoff $[8$, p. 112]. The second statement of the theorem follows from Lemma 1 preceding Theorem 1.2.

\section{Extensions of SPACES With ELEMENT 1}

Let $F$ be a $\sigma$-complete vector lattice with element 1 . As proved by Freudenthal [1], there is a subset $B$ of elements of $F$, between 0 and 1 , which form a $\sigma$-complete Boolean algebra, where the partial ordering of $B$ is the same as that of $F$. The subset $B$ is defined as follows: an element $e$ of $F$ belongs to $B$ if

$$
\wedge(e, 1-e)=0 .
$$

To an arbitrary element $a$ of $F$ can be made to correspond an element of $B$, namely the greatest lower bound of all elements of $B$ which are greater than or equal to $\wedge(1, a)$. This "inf" exists, since it can be computed as the countable "sup:"

$$
e(a)=\bigvee_{n=1}^{\infty} \wedge\left[(n a)^{+}, 1\right] .
$$

The following relation holds for any element $a$ of $F: e(a)+e(-a) \leqq 1$.

Now let $c$ be a fixed element of $F$. Then there is defined the function from real numbers to elements of $B$ :

$$
f(\alpha)=e_{\alpha}=e(c-\alpha) .
$$

Freudenthal proves that $c$ not only determines the function $f(\alpha)$, but that if $f(\alpha)$ is given by an element $c$, it in turn determines $c$, by a Lebesgue-Stieltjes integral representation.

Let $\Omega$ be the space of functions, as defined in Chapter I, determined by $B$. Considering $\Omega$ as a set of functions, we have

TheOREM 2.1. If $c \in F$, and $f(\alpha)=e(c-\alpha)$, then $f(\alpha) \in \Omega$.

Proof. We prove part two, observing first that the bounds indicated exist because of the monotoneity of $f(\boldsymbol{\alpha})$. 
Ad $2 \mathrm{~b}$ : We establish first the inequality $\wedge(m c-m n, n) \leqq c$, where $c \geqq 0$, and $m$ and $n$ are integers $\geqq 0$. Represent the left side of the inequality by $x$. Then $x \leqq n$, and therefore $-m n \leqq-m x$. Also $x \leqq m c-m n$ and therefore $x \leqq m c-m x$. That is, $(m+1) x \leqq m c \leqq(m+1) c$, or $x \leqq c$, as was to be proved. Now, since $0 \leqq c$,

$$
\vee[\wedge(m c-m n, n), 0] \leqq c,
$$

or, by the distributive law for $F$,

$$
\wedge[\vee(m c-m n, 0), n ! \leqq c .
$$

Therefore for every $m$ and $n \geqq 0$

$$
n \wedge\left[m(c-n)^{+}, 1\right]=\wedge\left[m n(c-n)^{+}, n\right] \leqq c,
$$

and so for every $n \geqq 0$

$$
n \bigvee_{m=1}^{\infty} \wedge\left[m(c-n)^{+}, 1\right]=n e(c-n) \leqq c .
$$

Therefore for any $c$ and for every integer $n \geqq 0$,

$$
n e(c-n) \leqq n e\left(c^{+}-n\right) \leqq c^{+} .
$$

Letting $e=\wedge_{n} e(c-n)$, we have $n e \leqq c^{+}$for every $n \geqq 0$. But this implies that $e=0$.

Ad 2a: We state without proof that for any $\alpha>0$ :

$$
e(-c)+e(c+\alpha) \geqq 1 \text {. }
$$

The proof is completed by application of part $2 \mathrm{~b}$, since for any positive number $n: e(-c-n)+e(c+2 n) \geqq 1$.

Notation. If $F$ is a $\sigma$-complete $F$-space, if $B$ is the Boolean algebra associated with $F$, and if $\Omega$ is the space defined in terms of $B$, then denote by $\Phi$ the subset of $\Omega$ consisting of all those functions which arise from elements of $F$ by the Freudenthal process. We shall denote this relationship:

$$
F \rightarrow \Phi \subseteq \Omega \text {. }
$$

The set $\Phi$ may be a proper subset of $\Omega$. For example, let the original $F$ be the space $L^{p}, p \geqq 1$, on the unit interval. Then the Boolean algebra, $B$ will be the algebra of equivalent classes of measurable sets, reduced modulo sets of measure zero. Let $f(x)$ be a fixed element of $L^{p}(c \in F)$. Then $e_{\alpha}$ will be the equivalence class corresponding to

$$
\underset{x}{E}[f(x)>\alpha]
$$

The space $\Omega$ will correspond to the space of all measurable functions, and will be a proper extension of $F$. 
Our problem now is to study the relation between the operations of the space $F$ and those of the space $\Omega$.

THEOREM 2.2. The space $\Omega$ is an extension of the space $F$. That is, the correspondence between the elements of $F$ and $\Phi$ is an isomorphism with respect to ordering, "sup," "inf," addition, and multiplication by real numbers.

Proof. We shall use the following notation: $a, b, c, d \in F ; f, g, h, k \in \Omega$; $a \rightarrow f$ means that $f$ is the function in $\Phi$ corresponding to $a$ in $F . \vee_{n}\left(a_{n}\right)$ is the $\bigvee$ in $F . \bigvee_{n}\left(f_{n}\right)$ is the $\vee$ in $\Omega$. (Similarly with $\wedge$, addition, and multiplication by real numbers.)

Lemma 1. Ordering is preserved.

If $c \rightarrow f, d \rightarrow g$, then $c \leqq d \rightarrow f \leqq g$. From the Freudenthal integral representation we conclude that $f \leqq g \rightarrow c \leqq d$. Combining these statements with the fact that $c=d \leftrightarrow f=g$, we have

$$
c<d \longleftrightarrow f<g .
$$

Lemma 2. Suprema correspond. That is, if $c_{n} \rightarrow f_{n}$, and if $\bigvee_{n} c_{n}$ exists and is equal to $c$, then $f=\bigvee_{n} f_{n}$ exists and

$$
c \rightarrow f \text {. }
$$

Since the $c_{n}$ are bounded above in $F$, and since order is maintained by the correspondence, the $f_{n}$ are bounded above in $\Phi$, and therefore in $\Omega$. Therefore $f=\bigvee_{n} f_{n}$ exists in $\Omega$. It remains to be proved that

Now

$$
\bigvee_{n=1}^{\infty} \bigvee_{m=1}^{\infty} \wedge\left[m\left(c_{n}-\alpha\right)^{+}, 1\right]=\bigvee_{m=1}^{\infty} \wedge\left[m\left(\underset{n}{\bigvee} c_{n}-\alpha\right)^{+}, 1\right]
$$

$$
\begin{aligned}
\wedge\left[m\left(\bigvee c_{n}-\alpha\right)^{+}, 1\right] & =\wedge\left[m \bigvee\left(\bigvee_{n} c_{n}-\alpha, 0\right), 1\right] \\
& =\wedge\left[m \underset{n}{\bigvee} \bigvee\left(c_{n}-\alpha, 0\right), 1\right]=\wedge\left[\bigvee_{n} m\left(c_{n}-\alpha\right)^{+}, 1\right]
\end{aligned}
$$

By the distributive law the last expression is equal to

$$
\bigvee_{n} \wedge\left[m\left(c_{n}-\alpha\right)^{+}, 1\right]
$$

and proof is completed by application of the associative law.

\section{Leмma 3. Finite infima correspond.}

It is sufficient to prove this for two elements. With a simplification of the notation, we wish to show that

$$
\wedge\left\{\bigvee_{n} \wedge\left(n c^{+}, 1\right), \bigvee_{n} \wedge\left(n d^{+}, 1\right)\right\}=\bigvee_{n} \wedge\left[n(\wedge(c, d))^{+}, 1\right]
$$


Because of the monotoneity of $\wedge\left(n c^{+}, 1\right)$ and $\wedge\left(n d^{+}, 1\right)$, the left side can be written $\vee_{n} \wedge\left\{\wedge\left(n c^{+}, 1\right), \wedge\left(n d^{+}, 1\right)\right\}$. Application of the distributive law completes the proof.

LEMMA 4. If $c \rightarrow f,-c \rightarrow g$, then $f+g \geqq 0$.

We wish to prove that for any $a$

$$
1-\bigvee_{n} \wedge\left[n(-a)^{+}, 1\right] \leqq \bigwedge_{m=1}^{\infty} \bigvee \wedge\left[n(a+1 / m)^{+}, 1\right]
$$

The left side is equal to

$$
\bigwedge_{n=1}^{\infty} \wedge[n \vee(a+1 / n, 0), 1]
$$

For any $m>0$, and $n \geqq m$ :

$$
\wedge[n \vee(a+1 / n, 0), 1] \leqq \wedge[n \vee(a+1 / m, 0), 1],
$$

and since the first term $\downarrow$ as $n \uparrow$,

$$
\begin{aligned}
\bigwedge_{n=1}^{\infty} \wedge[n \vee(a+1 / n, 0), 1] & =\bigwedge_{n>m} \wedge[n \vee(a+1 / n, 0), 1] \\
& \leqq \bigvee_{n=1}^{\infty} \wedge\left[n(a+1 / m)^{+}, 1\right]
\end{aligned}
$$

This is true for every $m$, and therefore the left side is less than or equal to

$$
\bigwedge_{m=1}^{\infty} \bigvee_{n=1}^{\infty} \wedge\left[n(a+1 / m)^{+}, 1\right]
$$

LEMMA 5. If $a \rightarrow f, b \rightarrow g, a+b \rightarrow h$, then $h \geqq f+g$.

The problem is to show that

$$
\begin{gathered}
\bigvee_{\rho} \wedge\left\{\bigvee_{n=1}^{\infty} \wedge\left[n(a-\rho)^{+}, 1\right], \bigvee_{n=1}^{\infty} \wedge\left[n(b+\rho-\alpha)^{+}, 1\right]\right\} \\
\leqq \bigvee_{n=1}^{\infty} \wedge\left[n(a+b-\alpha)^{+}, 1\right] .
\end{gathered}
$$

This will be accomplished if we can show that for any $a$ and $b$

$$
\wedge\left\{\bigvee_{n=1}^{\infty} \wedge\left(n a^{+}, 1\right), \bigvee_{n=1}^{\infty} \wedge\left(n b^{+}, 1\right)\right\} \leqq \bigvee_{n=1}^{\infty} \wedge\left[n(a+b)^{+}, 1\right]
$$

Because of the monotoneity of $\wedge\left(n a^{+}, 1\right)$ and $\wedge\left(n b^{+}, 1\right)$ this relation can be written 
$\bigvee_{n=1}^{\infty} \wedge\left[(n a)^{+},(n b)^{+}, 1\right] \leqq \bigvee_{n=1}^{\infty} \wedge\left[(n a+n b)^{+}, 1\right]$.

This will be proved if we can show that for any $a$ and $b$

$$
\wedge\left(a^{+}, b^{+}, 1\right) \leqq \wedge\left[(a+b)^{+}, 1\right] .
$$

This is true since

$$
\wedge\left(a^{+}, b^{+}\right) \leqq(a+b)^{+} .
$$

Proof of this statement is omitted.

Lemma 6. The zero elements of $F$ and $\Omega$ correspond.

Proof is omitted.

LEMMA 7. Negatives of corresponding elements correspond.

Suppose $c \rightarrow f,-c \rightarrow g$. Then by Lemma $4, f+g \geqq 0$. But $c+(-c)=0 \rightarrow 0$, and by Lemma $4,0 \geqq f+g$. Therefore $f+g=0$, or $-c \rightarrow-f$.

LeMma 8. Sums of corresponding elements correspond.

Let $a \rightarrow f, b \rightarrow g, a+b \rightarrow h$. Then by Lemma 7 ,

$$
-a \rightarrow-f,-b \rightarrow-g,-a+(-b)=-(a+b) \rightarrow-h .
$$

Therefore by Lemma $5, h \geqq f+g$, and $-h \geqq(-f)+(-g)=-(f+g)$, so that $h=f+g$.

LEMMA 9. Infima correspond.

Suppose $c_{n} \rightarrow f_{n}$. Then $-c_{n} \rightarrow-f_{n}$. Consequently

$$
\bigvee_{n}\left(-c_{n}\right) \rightarrow \bigvee_{n}\left(-f_{n}\right)
$$

and

$$
\bigwedge_{n}\left(c_{n}\right)=-\bigvee_{n}\left(-c_{n}\right) \rightarrow-\bigvee_{n}\left(-f_{n}\right)=\bigwedge_{n}\left(f_{n}\right) .
$$

LemMa 10. Multiplication by real numbers maintains correspondence.

Because of Lemma 7, and because multiplication by zero has already been considered, we need prove this only for positive numbers. But this is simple routine verification.

This completes the proof of Theorem 2.2.

If we apply the Freudenthal method of extracting a Boolean algebra from a $\sigma$-complete $F$-space to the space $\Omega$, we shall obtain precisely the characteristic functions. Therefore if we construct the space $\Omega$ a second time, we obtain no further extension. The space $\Omega$ is a maximal extension. 
We have already seen that the zero elements of the spaces $F$ and $\Omega$ correspond. The same is true of the elements 1 . In fact, we can state

THEOREM 2.3. The correspondence between the elements of the Boolean algebra $B$ and the characteristic functions of $\Omega$ coincides with the correspondence between the elements of $F$ and those of $\Phi$.

Proof is omitted.

Our statement regarding a maximal extension for any $\sigma$-complete $F$-space can now be worded:

Theorem 2.4. All spaces with the same Boolean algebra possess a common extension having the same Boolean algebra.

Freudenthal [1] states without proof that if $B$ is a $\sigma$-complete Boolean algebra, then a $\sigma$-complete $F$-space can be constructed having $B$ as its Boolean algebra. The statement is not made in terms of maximal extension, nor is the ring property mentioned, although a space having the properties of $\Omega$ was undoubtedly intended. Whether the construction of this space was to be similar to the present one, or more on the order of that of Carathéodory, we do not know.

\section{The SPACE $M$ OF Bounded Functions}

We consider in this chapter the analogue of the space of bounded measurable functions. It is easy to see that if $\Omega$ is a $\sigma$-complete $F$-space then the subspace $M$ of bounded functions is also a $\sigma$-complete $F$-space. But it is also a complete normed space. We introduce a norm first for the elements of $\mathrm{M}^{+}$.

Definition. If $f \in M^{+},\|f\|=1$. u.b. $\{\alpha\}$ where $f(\alpha)>0$.

Leмma. 1. $\|0\|=0$. If $f>0,\|f\|>0$. 2. If $0 \leqq f \leqq g,\|f\| \leqq\|g\|$. 3. If $f \geqq 0, \alpha \geqq 0$, then $\|\alpha f\|=\alpha\|f\|$. 4. If $f, g \geqq 0$, then $\|f+g\| \leqq\|f\|+\|g\|$.

Proof is omitted.

Definition. For arbitrary $f \in M,\|f\|=\||f|\|$.

Theorem 3.1. The space $M$ is normed.

Proof. 1. $\|f\| \geqq 0 .\|f\|=0 \longleftrightarrow f=0.2$. $\|f+g\| \leqq\|f\|+\|g\|$. The first is trivial and the second is true by the lemma above and because $|f+g| \leqq|f|+|g|$.

Theorem 3.2. The space $M$ is complete.

Proof. We first establish a lemma, using the notation $f_{n} \rightarrow^{\circ} f$ to represent (o)-convergence (cf. Birkhoff [8, chap. 2, §36]).

Lemma. If $f_{n}$ is a sequence of elements of $M^{+}$such that (1) $f_{n} \rightarrow^{\circ} 0$, (2) $\left\|f_{n}-f_{m}\right\| \rightarrow 0$, then

$$
\left\|f_{n}\right\| \rightarrow 0 \text {. }
$$


Proof of Lemma. By the assumption (2) above, given any $\beta>0$, we can find an $N$ such that for $n, m \geqq N$,

$$
\left(f_{n}-f_{m}\right)(\beta)=\bigvee_{\rho} \wedge\left[f_{n}(\rho), 1-\bigwedge_{p=1}^{\infty} f_{m}(-\beta+\rho-1 / p)\right]=0 .
$$

Because of the monotoneity of $f_{m}(-\beta+\rho-1 / p)$ as a function of $p$,

$$
\bigwedge_{p=1}^{\infty} f_{m}(-\beta+\rho-1 / p)=\bigwedge_{p>1 / \beta}^{\infty} f_{m}(-\beta+\rho-1 / p) \leqq f_{m}(-2 \beta+\rho),
$$

and therefore for any $\rho$

$$
\wedge\left[f_{n}(\rho), 1-f_{m}(-2 \beta+\rho)\right]=0 .
$$

In particular we may choose $\rho=3 \beta$, in which case we can state that for all $n, m \geqq N, \wedge\left[f_{n}(3 \beta), 1-f_{m}(\beta)\right]=0$. Therefore

$$
\bigvee_{m>N}^{\infty} \wedge\left[f_{n}(3 \beta), 1-f_{m}(\beta)\right]=\wedge\left[f_{n}(3 \beta), 1-\bigwedge_{m>N}^{\infty} f_{m}(\beta)\right]=0 .
$$

But since $\wedge_{m>N}^{\infty} f_{m}(\beta)=0$ by condition (1), $f_{n}(3 \beta)=0$ for all $n \geqq N$, and therefore $\left\|f_{n}\right\| \rightarrow 0$.

Before completing the proof of Theorem 3.2 we remark that, for an arbitrary sequence $f_{n}$ of elements of $M$,

$$
\left\|f_{n}\right\| \rightarrow 0 \rightarrow f_{n} \rightarrow^{\circ} 0
$$

but the reverse implication is not true.

Assuming we have a sequence $f_{n}$ of elements of $M$ such that

$$
\left\|f_{n}-f_{m}\right\| \rightarrow 0
$$

we wish to find an $f$ in $M$ such that

$$
\left\|f-f_{n}\right\| \rightarrow 0 .
$$

Following the remark above, we can state that

$$
f_{n}-f_{m} \rightarrow^{\circ} 0,
$$

and therefore, as proved by Kantorovitch [3], there exists an element $f$ of $M$ such that

$$
f-f_{n} \rightarrow^{\circ} 0 .
$$

We shall prove that

$$
\left\|f-f_{n}\right\| \rightarrow 0 .
$$
have

Let $F_{n}=f_{n}-f$. Then $\left|F_{n}\right| \rightarrow^{\circ} 0$. And, since $\left|F_{n}-F_{m}\right| \geqq|| F_{n}|-| F_{m}||$, we 
(1) $\left|F_{n}\right| \rightarrow^{\circ} 0$,

(2) ||$\left|F_{n}\right|-\left|F_{m}\right||| \rightarrow 0$.

Therefore, by the preceding lemma,

$$
\left\|\left|F_{n}\right|\right\|=\left\|F_{n}\right\|=\left\|f_{n}-f\right\| \rightarrow 0 .
$$

This completes the proof of Theorem 3.2.

We have seen that convergence according to ordering does not imply convergence according to the norm. Furthermore the space $M$ need not be regular (cf. Kantorovitch [3]). For example, the space of bounded measurable functions on the unit interval is not regular.

\section{THE $\Lambda^{p}$ SPACES}

In this chapter we shall be concerned with a Boolean algebra $B$ with a completely additive bounded measure.

Definition, $A$ Boolean algebra of type $B_{\mu}$ is a $\sigma$-complete Boolean algebra $B$ with a real-valued (measure) function $\mu(a)$ defined for every element $a$ of $B$ and such that

1. $0 \leqq \mu(a) \leqq 1$ for every $a$ of $B$,

2. $\mu(a)=0 \longleftrightarrow a=0$,

3. $\mu(1)=1$,

4. if $\wedge(a, b)=0, \mu \bigvee(a, b)=\mu(a)+\mu(b)$,

5. if $a_{n} \downarrow 0, \mu a_{n} \downarrow 0$.

Remarks. 1. If $a_{n} \downarrow a, \mu a_{n} \downarrow \mu a$; if $a_{n} \uparrow a, \mu a_{n} \uparrow \mu a .2$. If $\left(a_{1}, a_{2}, \cdots\right)$ is any non-empty countable set of elements of $B$, then

$$
\mu \vee\left(a_{1}, a_{2}, \cdots\right) \leqq \mu\left(a_{1}\right)+\mu\left(a_{2}\right)+\cdots .
$$

If the elements are pairwise disjoint we have equality.

Theorem 4.1. A Boolean algebra $B$ of type $B_{\mu}$ is complete. In fact, if $F$ is any subset of $B$, then there is a countable subset $F^{\prime} \subseteq F$ such that $\vee\left(F^{\prime}\right)=\bigvee(F)$.

Proof is given by Wecken [7].

In this section we construct spaces similar to the Lebesgue $L^{p}$ spaces and belonging to the class of what Kantorovitch [3] calls spaces of type $B_{2}$, which we shall call normed $\dot{K}$-spaces. They are defined:

Definition. $A$ normed $K$-space is a $\sigma$-complete $K$-space $K$ with a norm $\|f\|$ satisfying the following conditions for the elements of $K^{+}$:

1. $\|0\|=0 ;\|f\|>0$ if $f>0$;

2. $\|f+g\| \leqq\|f\|+\|g\|$;

3. $\|\alpha f\|=\alpha\|f\|$ where $\alpha>0$;

4. if $f<g$, then $\|f\|<\|g\|$;

5. if $f_{n} \downarrow 0$, then $\left\|f_{n}\right\| \downarrow 0$; if $f_{n} \uparrow \infty$, then $\left\|f_{n}\right\| \uparrow \infty$. 
For arbitrary elements, the norm is given by

$$
\|f\|=\||f|\| \text {. }
$$

Definition. $A$ normed $F$-space is a $\sigma$-complete $F$-space, where the element 1 has norm equal to 1.

Definition. $A$ space of type $K^{p}\left(F^{p}\right), p \geqq 1$, is a $\sigma$-complete $K$-space (F-space) such that $\wedge(f, g)=0 \rightarrow\|f+g\|^{p}=\|f\|^{p}+\|g\|^{p}$.

We shall discover a close analogy between spaces of type $F^{p}$ and the Lebesgue $L^{p}$ spaces. A similar problem, from the axiomatic viewpoint, has been studied by Bohnenblust.

Let us reverse the direction of investigation for a moment and consider the Boolean algebra of a space of type $F^{p}, p \geqq 1$, when the latter is given. Define the function on the elements of $B$ :

$$
\mu(a)=\|a\| p .
$$

Then it is easily seen that the Boolean algebra is of type $B_{\mu}$.

Returning to the main problem, let $B$ be a Boolean algebra of type $B_{\mu}$, and let $\Omega$ be the space of functions on $B$. Let $p$ be any fixed real number $\geqq 1$, and consider the elements of $\Omega^{+}$.

Definition. If $s$ is the simple function

$$
s=\alpha_{1} a_{1}+\cdots+\alpha_{n} a_{n},
$$

where $\alpha_{i} \geqq 0, i=1,2, \cdots, n$, and $\wedge\left(a_{i}, a_{j}\right)=0, i \neq j$, then we define its integral:

$$
\int s d \mu=\alpha_{1} \mu a_{1}+\cdots+\alpha_{n} \mu a_{n}
$$

and its norm:

$$
\|s\|=\left[\int s^{p} d \mu\right]^{1 / p}=\left[\alpha_{1}^{p} \mu a_{1}+\cdots+\alpha_{n}^{p} \mu a_{n}\right]^{1 / p} .
$$

For an arbitrary $f \in \Omega^{+}$we define the integral:

$$
\int f d \mu=\sup \left\{\int s d \mu\right\} \text { for all simple functions } s, \quad 0 \leqq s \leqq f .
$$

If we denote by $M^{p}$ the set of all $f \in \Omega^{+}$such that $\int f^{p} d \mu<\infty$, we can define the norm of $f$, for any $f \in M^{p}$ :

$$
\|f\|=\left[\int f^{p} d \mu\right]^{1 / p} .
$$

In order to prove that this norm satisfies the necessary conditions, we establish a number of lemmas: 
Lemma 1. If $g \in M^{p}, 0 \leqq f \leqq g$, then $f \in M^{p}$ and $\|f\| \leqq\|g\|$.

Proof is trivial. In particular, every bounded function of $\Omega^{+}$belongs to $M^{p}$ for every $p \geqq 1$.

Lemma 2. If $s$ and $t$ are simple functions, $\geqq 0$, then

$$
\begin{gathered}
\int(s+i) d \mu=\int s d \mu+\int t d \mu, \\
\|s+t\| \leqq\|s\|+\|t\| .
\end{gathered}
$$

Proof rests on the fact that $s$ and $t$, and therefore $s+t$, can be represented as linear combinations, with non-negative coefficients, of the same set of pairwise disjoint characteristic functions. The second relation follows from an application of Minkowski's inequality.

In order to extend these relations to arbitrary functions in $M^{p}$, we prove

LEMMA 3. If $\left\{s_{n}\right\}$ is an increasing sequence of positive simple functions whose limit is the function $f$, then

$$
\int s_{n} d \mu \uparrow \int f d \mu,
$$

whether the limit be finite or infinite.

Proof. If $s$ is any simple function such that $0 \leqq s \leqq f$, and if $\alpha>0$, we wish to find an $n$ such that

$$
\int s_{n} d \mu>\int s d \mu-\alpha
$$

The method of obtaining $n$ is straightforward.

From this lemma follows immediately:

LemMA 4. If $f \in M^{p}$, and if $0 \leqq s_{n} \uparrow f$, where $s_{n}$ are simple functions, then $\left\|s_{n}\right\| \uparrow\|f\| . A l s o$

$$
\|f\|=\sup \{\|s\|\}
$$

for all simple functions $s$ such that $0 \leqq s \leqq f$.

In the proof of Theorem 1.12 an increasing sequence of simple functions was given having as limit a given function of $\Omega^{+}$. From the form of those simple functions and from Lemma 3 we have

Lemma 5. If $f \in \Omega^{+}$, then $\int f d \mu$, finite or infinite, is equal to the least upper bound of sums of the form:

$$
\alpha_{1} \mu f\left(\alpha_{1}\right)+\left(\alpha_{2}-\alpha_{1}\right) \mu f\left(\alpha_{2}\right)+\cdots+\left(\alpha_{n}-\alpha_{n-1}\right) u f\left(\alpha_{n}\right),
$$

where $0 \leqq \alpha_{1} \leqq \cdots \leqq \alpha_{n}$. 
Similarly we have

Lemma 6. If $f \in M^{p}$, then $\|f\|^{p}$ is equal to the least upper bound of sums of the form:

$$
\alpha_{1}^{p} \mu f\left(\alpha_{1}\right)+\cdots+\left(\alpha_{n}^{p}-\alpha_{n-1}^{p}\right) \mu f\left(\alpha_{n}\right),
$$

where $0 \leqq \alpha_{1} \leqq \cdots \leqq \alpha_{n}$.

TheOREM 4.2. If $f, g \in M^{p}, p \geqq 1$, then $f+g \in M^{p}$ and

$$
\|f+g\| \leqq\|f\|+\|g\| \text {. }
$$

Proof. Let $0 \leqq s_{n} \uparrow f, 0 \leqq t_{n} \uparrow g$. Then $0 \leqq s_{n}+t_{n} \uparrow f+g$ and therefore $\left\|s_{n}+t_{n}\right\| \uparrow\|f+g\|$. But $\left\|s_{n}+t_{n}\right\| \leqq\left\|s_{n}\right\|+\left\|t_{n}\right\| \leqq\|f\|+\|g\|$ for every $n$. The inequality follows.

Theorem 4.3. If $f, g \in M^{1}$, then $f+g \in M^{1}$ and $\|f+g\|=\|f\|+\|g\|$.

Proof. Let $0 \leqq s_{n} \uparrow f, 0 \leqq t_{n} \uparrow g$; and given $\alpha>0$, choose $n$ so that $\int f d \mu<\int s_{n} d \mu+\alpha, \int g d \mu<\int t_{n} d \mu+\alpha$. Then

$$
\int(f+g) d \mu \geqq \int\left(s_{n}+t_{n}\right) d \mu>\int f d \mu+\int g d \mu-2 \alpha .
$$

The implied inequality combined with that of the previous theorem, gives the desired equality.

LEMma. If $\wedge(a, b)=0$, then

$$
f_{a} \in M^{p}, f_{b} \in M^{p} \leftrightarrow f \vee_{(a, b)} \in M^{p}
$$

and

$$
\left\|f \vee_{(a, b)}\right\|^{p}=\left\|f_{a}\right\|^{p}+\left\|f_{b}\right\|^{p} .
$$

Proof. We may assume $p=1$, and proof follows from Theorem 4.3.

Theorem 4.4. If $\wedge(f, g)=0$, then $f, g \in M^{p}$ if and only if $f+g \in M^{p}$, and $\|f+g\|^{p}=\|f\|^{p}+\|g\|^{p}$.

Proof. In the preceding lemma set $a=f(0), b=g(0)$.

Definition. Let $\Lambda^{p}$ be the subspace of $\Omega$ consisting of all $f$ such that $|f| \in M^{p}$. If $f \in \Lambda^{p}$, we define the norm of $f$ :

$$
\|f\|=\||f|\| \text {. }
$$

If $f \in \Lambda^{1}$, we define the integral of $f$ :

$$
\int f d \mu=\int f^{+} d \mu-\int f^{-} d \mu .
$$

Remarks. 1. $M^{p}=\left[\Lambda^{p}\right]^{+}, 2$. if $1 \leqq p \leqq q, \Lambda^{q} \subseteq \Lambda^{p}$. 
Theorem 4.5. If $f, g \in \Lambda^{1}$, then $f+g \in \Lambda^{1}$, and

$$
\int(f+g) d \mu=\int f d \mu+\int g d \mu .
$$

Proof. Since $|f+g| \leqq|f|+|g|, f+g \in \Lambda^{1}$. Applying the additivity of the integral for elements of $\left[\Lambda^{1}\right]+$ to the known equality $(f+g)^{+}+f^{-}+g^{-}=(f+g)^{-}$ $+f^{+}+g^{+}$, and rearranging terms, we have the desired relation:

$$
\int(f+g)^{+} d \mu-\int(f+g)^{-} d \mu=\int f^{+} d \mu-\int f^{-} d \mu+\int g^{+} d \mu-\int g^{-} d \mu .
$$

We now state the fundamental theorem of this section:

Theorem 4.6. $\Lambda^{p}$ is a space of type $F^{p}, p \geqq 1$.

Proof. 1. If $f, g \in \Lambda^{p}$, then $f+g \in \Lambda^{p}$, since $|f+g| \leqq|f|+|g|$; 2. if $f \in \Lambda^{p}, \alpha$ real, then $\alpha f \in \Lambda^{p}$, since $|\alpha f|=|\alpha||f| ; 3$. if $f \in \Lambda^{p}$, then $f^{+} \in \Lambda^{p}$, since $f^{+} \leqq|f| ; 4$. if $f \leqq g \leqq h, f, h \in \Lambda^{p}$, then $g \in \Lambda^{p}$, since $|g| \leqq h^{+}+f^{-} ; 5 .\|1\|=1$.

Therefore $\Lambda^{p}$ is a $\sigma$-complete $F$-space. We now verify the five conditions of the definition of a normed $K$-space.

Ad 1, 2, 3: Already proved, or trivial.

Ad 4: If $0 \leqq f<g$, then $0 \leqq f^{p}<g^{p}$, and therefore

$$
\|f\|^{p}=\int f^{p}<\int g^{p}=\|g\|^{p} .
$$

Ad 5: We state without proof the lemma:

LEMmA. If $f_{n} \geqq 0, g \geqq 0$ are elements of a vector lattice, and if $f_{n} \downarrow$, then $f_{n}-\wedge\left(f_{n}, g\right) \downarrow$.

Assume that $f_{n} \downarrow 0$, and that $\alpha$ is any number $>0$. We can find an $N$ such that $\int\left[f_{1}-\wedge\left(f_{1}, N\right)\right] d \mu<\alpha$. Then for everry $n \int\left[f_{n}-\wedge\left(f_{n}, N\right)\right] d \mu<\alpha$. In order to make $\int f_{n} d \mu<2 \alpha$, we wish to find an $n$ such that $\int \wedge\left(f_{n}, N\right) d \mu<\alpha$. An $n$ which will accomplish this will be any one such that

$$
\mu\left[f_{n}(\alpha / 2)\right]<\alpha / 2 N .
$$

Now assume that $0 \leqq f_{n} \uparrow \infty$, and that $\left\|f_{n}\right\| \leqq M$ for every $n$. We shall obtain a contradiction. Define

$$
f(\xi)=\bigvee_{n} f_{n}(\xi)
$$

Then $f(\xi)$ must certainly belong to $\Omega$, or we easily obtain a contradiction. Now form the sum, for $0<\beta_{1}<\cdots<\beta_{n}$ :

$$
\beta_{1} \mu f\left(\beta_{1}\right)+\cdots+\left(\beta_{n}-\beta_{n-1}\right) \mu f\left(\beta_{n}\right) .
$$


Given $\alpha>0$, choose $k_{1}, \cdots, k_{n}$ such that

$$
\begin{aligned}
& \mu\left[f_{k_{1}}\left(\beta_{1}\right)\right]>\mu\left[f\left(\beta_{1}\right)\right]-\alpha / \beta_{n}, \\
& \mu\left[f_{k_{n}}\left(\beta_{n}\right)\right]>\mu\left[f\left(\beta_{n}\right)\right]-\alpha / \beta_{n} .
\end{aligned}
$$

Letting $N=\max \left(k_{1}, \cdots, k_{n}\right)$, we have

is less than

$$
\beta_{1} \mu f\left(\beta_{1}\right)+\cdots+\left(\beta_{n}-\beta_{n-1}\right) \mu f\left(\beta_{n}\right)
$$

$$
\begin{aligned}
\beta_{1}\left[\mu f_{N}\left(\beta_{1}\right)+\alpha / \beta_{n}\right] & +\cdots+\left(\beta_{n}-\beta_{n-1}\right)\left[\mu f_{N}\left(\beta_{n}\right)+\alpha / \beta_{n}\right] \\
& \leqq \beta_{1 \mu f_{N}}\left(\beta_{1}\right)+\cdots+\left(\beta_{n}-\beta_{n-1}\right) \mu f_{N}\left(\beta_{n}\right)+\frac{\alpha}{\beta_{n}} \beta_{n} \leqq M+\alpha .
\end{aligned}
$$

Therefore $f \in \Lambda^{p}$. (Contradiction.) This completes the proof of Theorem 4.6.

Remark. As proved by Kantorovitch [3], a normed $K$-space is regular, and convergence according to the norm is the same as star-convergence. This statement is therefore true for the $\Lambda^{p}$ spaces. Let us observe also that the simple functions are dense, in the sense of the norm, in $\Lambda^{p}$ for every $p \geqq 1$.

THEOREM 4.7. Every space of type $F^{p}, p \geqq 1$, is isomorphic to the space $\Lambda^{p}$, where the basic Boolean algebra of $\Lambda^{p}$ is the Boolean algebra of $F^{p}$.

Proof. We have seen that the Boolean algebra of $F^{p}$ is of type $B_{\mu}$, and that therefore the corresponding spaces $\Omega$ and $\Lambda^{p}$ can be constructed. As proved in the second chapter, the space $F^{p}$ is isomorphic to a subspace of $\Omega$. This subspace is precisely the space $\Lambda^{p}$. The isomorphism preserves norm. Details of the proof are omitted.

\section{Absolute continuity}

In this chapter we shall study the space $A C$ of additive absolutely continuous real-valued functions defined on a Boolean algebra of type $B_{\mu}$, and set up an isomorphism between $A C$ and $\Lambda^{1}$.

Definition. $A$ real-valued function, $\xi(a)$, defined on the elements of a Boolean algebra $B$, of type $B_{\mu}$, is absolutely continuous if, given any $\alpha>0$, there exists a $\beta>0$ such that

$$
\mu(a)<\beta \rightarrow|\xi(a)|<\alpha .
$$

Definition. The space $A C$ is the space of all additive absolutely continuous real-valued functions, $\xi(a)$, defined on the elements of a Boolean algebra $B$ of type $B_{\mu}$, with the usual definitions of addition, multiplication by real numbers, and ordering.

We shall show that $A C$ is a space of type $F^{1}$ by obtaining an isomorphism 
between $A C$ and $\Lambda^{1}$. First we set up a one-to-one correspondence between $A C^{+}$and $\left[\Lambda^{1}\right]^{+}$.

Definition. We define the integral of a function, $f \in \Lambda^{1}$, over an element of the Boolean algebra $B$ :

$$
\int_{a} f d \mu \equiv \int f_{a} d \mu .
$$

DEFINITION: The transformation $X$ is defined by the equation

$$
\xi(\boldsymbol{a}) \equiv X[f(\xi)]
$$

where $f \in\left[\Lambda^{1}\right]+$ and $\xi(a) \equiv \int_{a} f d \mu$. The transformation $X$ is an integration operation. We say that $\xi(\boldsymbol{a})$ is the indefinite integral of $f(\xi)$.

Theorem 5.1. If $f \in\left[\Lambda^{1}\right]^{+}$, then $X(f) \in A C^{+}$.

Proof. Positiveness and finite additivity are easily verified. Let $\alpha>0$, and let $f=f_{1}+f_{2}$, where $f_{1}$ and $f_{2}$ belong to $\left[\Lambda^{1}\right]^{+}, f_{1}$ is bounded by $N$, and $\int f_{2} d \mu<\alpha$. Then

$$
\int_{a} f d \mu<\int_{a} f_{1} d \mu+\alpha .
$$

Now, if $\mu(a)<N / \alpha$, we have $\xi(a)=\int_{a} f d \mu<2 \alpha$.

Theorem 5.2. Let $\xi(a) \in A C^{+}$and let $\alpha \geqq 0$. Form the set $S_{\alpha}$ consisting of the zero of $B$ and all $a$ of $B$ such that

$$
0<b \leqq a \rightarrow \xi(b)>\alpha \cdot \mu(b) .
$$

Then $S_{\alpha}$ is a principal ideal. That is, if

$$
a \leqq \lambda(\alpha)=\bigvee\left(S_{\alpha}\right)
$$

then $a \in S_{\alpha}$.

Proof. 1. If $a_{1} \in S_{\alpha}, a_{2} \leqq a_{1}$, then $a_{2} \in S_{\alpha}$. 2. If $a_{1}, a_{2} \in S_{\alpha}$, then $\bigvee\left(a_{1}, a_{2}\right) \in S_{\alpha}$. 3. If $a_{1}, a_{2}, \cdots \in S_{\alpha}$, then $\vee\left(a_{1}, a_{2}, \cdots\right) \in S_{\alpha}$. 4. If $Q \subseteq S_{\alpha}$, then $\vee(Q) \in S_{\alpha}$.

Ad 2: We may assume $a_{1}$ and $a_{2}$ to be disjoint. Then if $0<b \leqq \bigvee\left(a_{1}, a_{2}\right)$, write $b=\bigvee\left(b_{1}, b_{2}\right)$, where $b_{1}=\wedge\left(b, a_{1}\right), b_{2}=\wedge\left(b, a_{2}\right)$. Then $\xi\left(b_{1}\right) \geqq \alpha \mu\left(b_{1}\right)$, $\xi\left(b_{2}\right) \geqq \alpha \mu\left(b_{2}\right)$, where the strict > relation must hold in at least one case since not both $b_{1}$ and $b_{2}$ can be zero. Therefore $\xi(b)>\alpha \mu(b)$.

Ad 4: In a Boolean algebra of type $B_{\mu}$ any "sup" can be reduced to a countable one.

Definition. The transformation $Y$ is defined by the equation

$$
f(\xi) \equiv Y[\xi(a)]
$$


where $\xi(a) \in A C^{+}$and

$$
f(\xi) \equiv\left\{\begin{array}{cc}
1, & \xi<0, \\
\vee\left(S_{\xi}\right), & \xi \geqq 0 .
\end{array}\right.
$$

The transformation $Y$ is a differentiation operation. We say that $f(\xi)$ is the derivative of $\xi(\boldsymbol{a})$.

THEOREM 5.3. If $f \in\left[\Lambda^{1}\right]^{+}$, then $Y[X(f)]=f$. That is, the derivative of the integral of a function $f \in\left[\Lambda^{1}\right]+$ is $f$.

Proof. Given any $\alpha \geqq 0$, we wish to show :

1. $f(\alpha) \in S_{\alpha}$.

2. $a \in S_{\alpha} \rightarrow a \leqq f(\alpha)$.

Ad 2: Assume there exists an $a>0$ such that the inequality $a \leqq f(\alpha)$ does not hold, $0<b \leqq a \rightarrow \int_{b} f d \mu>\alpha \mu(b)$. But if we take $b=a-\wedge[a, f(\alpha)]$, then $0<b \leqq a$, and therefore $\int_{b} f d \mu>\alpha \mu(b)$. This is absurd since $f_{b}(\alpha)=\wedge[b, f(\alpha)]=0$. The case $S_{\alpha}=0$ is trivial.

TheOREM 5.4. Let $\xi(a) \in A C^{+}, \alpha \geqq 0$, and let $S_{\alpha}$ be the principal ideal defined above. If $a=\bigvee\left(S_{\alpha}\right), \wedge(a, c)=0$, then $\xi(c) \leqq \alpha \mu(c)$.

Proof. Suppose $0<c, \wedge(a, c)=0, \xi(c)>\alpha \mu(c)$. Let $\beta_{n} \downarrow 0$.

Let $m_{1}$ be the least upper bound of $\mu(d)$ for all $d<c$ such that $\xi(d) \leqq \alpha \mu(d)$. Then $m_{1}>0$. Find $c_{1}$ such that

$$
0<c_{1}<c, \quad \xi\left(c_{1}\right) \leqq \alpha \mu\left(c_{1}\right), \quad \mu\left(c_{1}\right)>m_{1}-\beta_{1} .
$$

In general let $m_{n}>0$ be the least upper bound of $\mu(d)$ for all $d$ such that $d<c-c_{1}-\cdots-c_{n-1} ; \xi(d) \leqq \alpha \mu(d)$. Pick $c_{n}$ such that

$$
0<c_{n}<c-c_{1}-\cdots-c_{n-1}, \quad \xi\left(c_{n}\right) \leqq \alpha \mu\left(c_{n}\right), \quad \mu\left(c_{n}\right)>m_{n}-\beta_{n} .
$$

Regarding the measures, we must have $m_{n} \leqq \beta_{n-1}, n \geqq 2$. For suppose there is an $n$ such that $m_{n}>\beta_{n-1}$. Then find an element $b<c-c_{1}-\cdots-c_{n-1}$ such that $\mu(b)>\beta_{n-1}$ and $\xi(b) \leqq \alpha \mu(b)$. Then $\mu\left(c_{n-1}+b\right)>m_{n-1}-\beta_{n-1}+\beta_{n-1}=m_{n-1}$. But since $c_{n-1}+b<c-c_{1}-\cdots-c_{n-2}$, and $\xi\left(c_{n-1}+b\right) \leqq \alpha \mu\left(c_{n-1}+b\right)$, we know that $\mu\left(c_{n-1}+b\right) \leqq m_{n-1}$. (Contradiction.)

Form $c^{\prime}=\bigvee_{n=1}^{\infty} c_{n} \leqq c$. Then by absolute continuity, $\xi\left(c^{\prime}\right) \leqq \alpha \mu\left(c^{\prime}\right)$, and therefore $c^{\prime}<c$ and $\xi\left(c-c^{\prime}\right)>\alpha \mu\left(c-c^{\prime}\right)$. Therefore there exists a $d$ such that $0<d<c-c^{\prime}, \xi(d) \leqq \alpha \mu(d)$.

Now choose an $n$ such that $\beta_{n-1}<\mu(d)$. Then $m_{n}<\mu(d)$. Therefore, since $d<c-c_{1}-\cdots-c_{n-1}, \mu(d) \leqq m_{n}$. (Contradiction.)

Theorem 5.5. If $\xi(a) \in A C^{+}$, then $f(\xi) \equiv Y[\xi(a)] \in\left[\Lambda^{1}\right]^{+}$.

Proof. We shall first verify that $f \in \Omega^{+}$. It is sufficient to verify parts $1,2 \mathrm{~b}$, and 3 in the definition of $\Omega$. 
Ad 1: If $0 \leqq \alpha<\beta, S_{\beta} \subseteq S_{\alpha}, f(\beta) \leqq f(\alpha)$.

Ad 2b: Suppose that for every $n=1,2, \cdots$

$$
\vee\left(S_{n}\right) \geqq d>0 \text {. }
$$

Then $d \in S_{n}$ for every $n$, and therefore $\xi(d)>n \mu(d)$ for every $n$. But this is absurd.

Ad 3: We wish to prove that for any $\alpha \geqq 0$

$$
\bigvee_{n=1}^{\infty} \bigvee\left(S_{\alpha+1 / n}\right)<\bigvee\left(S_{\alpha}\right)
$$

leads to a contradiction. Obviously the right side is $>0$. Let $b_{n}=\vee\left(S_{\alpha}\right)$ $-\bigvee\left(S_{\alpha+1 / n}\right)$. Then $b_{n} \downarrow b>0$. Therefore $\xi(b)>\alpha \mu(b)$. Pick an $M$ such that $\xi(b)$ $>(\alpha+1 / M) \mu(b)$. Then there exists an $N>M$ such that $\xi(b)>(\alpha+1 / M) \mu\left(b_{N}\right)$, so that $\xi\left(b_{N}\right)>(\alpha+1 / N) \mu\left(b_{N}\right)$. Since $\wedge\left[b_{N}, \vee\left(S_{\alpha+1 / N}\right)\right]=0$, the necessary contradiction is provided by Theorem 5.4.

In verifying that $f(\xi) \in\left[\Lambda^{1}\right]^{+}$, we shall prove more. Let $a$ be an arbitrary positive element of $B$, and, for an arbitrary set of real numbers, $\alpha_{1}<\alpha_{2}<\cdots<\alpha_{n}$, form

$$
\alpha_{1} \mu \wedge\left[a, f\left(\alpha_{1}\right)\right]+\cdots+\alpha_{n}-\left(\alpha_{n-1}\right) \mu \wedge\left[a, f\left(\alpha_{n}\right)\right] .
$$

Defining $b_{i}=\wedge\left[a, f\left(\alpha_{i}\right)\right]$, we can write this sum:

$$
\alpha_{1} \mu\left(b_{1}-b_{2}\right)+\alpha_{2} \mu\left(b_{2}-b_{3}\right)+\cdots+\alpha_{n} \mu\left(b_{n}\right) .
$$

Since $b_{i}-b_{i+1} \leqq b_{i} \leqq f\left(\alpha_{i}\right)$, we have $\xi\left(b_{i}-b_{i+1}\right) \geqq \alpha_{i} \mu\left(b_{i}-b_{i+1}\right)$, where the equality holds only if $b_{i}=b_{i+1}$. Therefore the previous sum is less than or equal to

$$
\xi\left(b_{1}-b_{2}\right)+\xi\left(b_{2}-b_{3}\right)+\cdots+\xi\left(b_{n-1}-b_{n}\right)+\xi\left(b_{n}\right)=\xi\left(b_{1}\right) \leqq \xi(a) .
$$

Therefore $\int_{a} f d \mu=\int f_{a} d \mu \leqq \xi(a)$. The proof of the theorem is completed when we set $a=1$.

The last inequality above implies the

Lemma. If $\xi(a) \in A C^{+}$, then $X[Y(\xi(a))] \leqq \xi(a)$.

The inequality can be replaced by an equality:

Theorem 5.6. If $\xi(a) \in A C^{+}$, then $X[Y(\xi(a))]=\xi(a)$. That is, every additive absolutely continuous function $\xi(a) \geqq 0$ is an integral. It is equal to the integral of its derivative.

Proof. We shall establish this theorem by means of lemmas:

Lemma 1. If $h=f_{a}$, and if $\alpha \geqq 0$, then $h(\alpha)=\vee\left(S_{\alpha, a}\right)$, where $S_{\alpha, a}$ is the set consisting of zero and all positive elements $c$ of $B$ such that $c \leqq a$, and $0<b \leqq c \rightarrow \xi(b)>\alpha \mu(b)$.

Proof is trivial. 
LEMMA 2. If $\alpha \geqq 0, \beta \geqq 0$, then

$$
\alpha \mu[h(\alpha)-h(\alpha+\beta)] \leqq \xi[h(\alpha)-h(\alpha+\beta)] \leqq(\alpha+\beta) \mu[h(\alpha)-h(\alpha+\beta)] .
$$

Proof. The first inequality follows from the fact that $h(\alpha)-h(\alpha+\beta) \leqq h(\alpha)$. The second inequality is obvious if $h(\alpha+\beta)=h(\alpha)$. Assume $h(\alpha+\beta)<h(\alpha)$. Then $0<h(\alpha)-h(\alpha+\beta) \leqq a, \wedge[h(\alpha)-h(\alpha+\beta), h(a+\beta)]=0$, and the inequality is a consequence of Theorem 5.4.

This result can be restated as

LEMMA 3. If $\rho>0, \alpha \geqq 0$, then for $0 \leqq \beta \leqq \rho$,

$0 \leqq \xi[h(\alpha)-h(\alpha+\beta)]-\alpha \mu[h(\alpha)-h(\alpha+\beta)] \leqq \rho \mu[h(\alpha)-h(\alpha+\beta)]$.

LemMA 4. Let $C=\bigvee\left(S_{\alpha}\right), A=\bigvee\left(S_{\alpha, a}\right)=\wedge(a, C)$. If $\wedge(A, b)=0, b \leqq a$, then $\xi(b) \leqq \alpha \mu(b)$.

Proof. Since $\wedge(A, b)=\wedge(a, b, C)=\wedge(b, C)=0$, we can apply Theorem 5.4 .

Lemma 5. Given $\rho<0$, we can find $\alpha_{1}<\alpha_{2}<\cdots<\alpha_{n}$ such that

$$
\xi(a)-\left[\alpha_{1} \mu h\left(\alpha_{1}\right)+\cdots+\left(\alpha_{n}-\alpha_{n-1}\right) \mu h\left(\alpha_{n}\right)\right]<3 \rho .
$$

Proof. By Lemma 4, and since $h(0)=\bigvee\left(S_{0, a}\right)$, we have $\xi[a-h(0)]=0$, and therefore we can pick $\alpha_{1}$ such that

$$
\xi(a)-\xi\left[h\left(\alpha_{1}\right)\right]<\rho .
$$

Choose $N>\alpha_{1}$ such that $\xi[h(N)]<\rho$, and finally choose $\alpha_{1}$ (as above) $<\alpha_{2}<\cdots<\alpha_{n}=N$ such that $\alpha_{i}-\alpha_{i-1}<\rho, i=2, \cdots, n$. Then

$$
\begin{aligned}
\xi(a)-\left[\alpha _ { 1 } \mu \left(h\left(\alpha_{1}\right)-\right.\right. & \left.\left.h\left(\alpha_{2}\right)\right)+\cdots+\alpha_{n} \mu h\left(\alpha_{n}\right)\right] \\
= & \xi\left[a-h\left(\alpha_{1}\right)\right]+\xi\left[h\left(\alpha_{1}\right)-h\left(\alpha_{2}\right)\right]+\cdots+\xi\left[h\left(\alpha_{n}\right)\right] \\
& -\alpha_{1} \mu\left[h\left(\alpha_{1}\right)-h\left(\alpha_{2}\right)\right]-\cdots-\alpha_{n} \mu h\left(\alpha_{n}\right) \\
\leqq & \rho+\rho\left[\mu\left(h\left(\alpha_{1}\right)-h\left(\alpha_{2}\right)\right)+\cdots+\mu\left(h\left(\alpha_{n-1}\right)-h\left(\alpha_{n}\right)\right)\right]+\rho \\
= & 2 \rho+\rho\left[\mu\left(h\left(\alpha_{1}\right)-h\left(\alpha_{n}\right)\right)\right] \leqq 3 \rho .
\end{aligned}
$$

This completes the proof of Theorem 5.6.

We shall now consider arbitrary elements of $A C$.

TheOREM 5.7. Let $\xi(a) \in A C$, and let $S$ be the set consisting of zero and all positive elements $a$ of $B$ such that

$$
0<b \leqq a \rightarrow \xi(b)>0 .
$$

Then $S$ is a principal ideal.

Proof. Proof is similar to that of Theorem 5.2.

Theorem 5.8. If $A=\bigvee(S), \wedge(c, A)=0$, then $\xi(c) \leqq 0$. 
Proof is similar to that of Theorem 5.4.

As before, we can define $S_{a}$ to be the set consisting of all elements of the form $\wedge(a, d)$ where $d \in S$. Then $S_{a}$ is also a principal ideal. We can also establish the

Lemma. Let $C=\vee(S), A=\bigvee\left(S_{a}\right)=\wedge(a, C)$. If $\wedge(A, b)=0$, and $b \leqq a$, then $\xi(b) \leqq 0$.

Definition. Given any $\xi(a) \in A C$ and any $a \in B$, let $a^{+}=\bigvee\left(S_{a}\right)$. (Then $a^{+}$ is a function of $\xi(\mathbf{a})$ and a.) Define $\xi^{+}(\mathbf{a}), \xi^{-}(\mathbf{a})$ :

$$
\xi^{+}(a) \equiv \xi\left(a^{+}\right), \quad \xi^{-}(a) \equiv-\xi\left(a-a^{+}\right) .
$$

Theorem 5.9. If $\xi(a) \in A C$, then $\xi^{+}(a)$ and $\xi^{-}(a) \in A C$ and $\xi^{+}(a)=\vee[\xi(a)$, $0(a)], \xi^{-}(a)=\vee[-\xi(a), 0(a)]$.

Proof is omitted.

The space $A C$ is therefore a vector lattice.

We proceed now to the establishment of the one-to-one correspondence between the elements of $A C$ and those of $\Lambda^{1}$. We extend the definitions of $X$ and $Y$ :

Definition. Given any element $f \in \Lambda^{1}$, we define

$$
\xi(\mathbf{a}) \equiv X(f) \equiv X\left(f^{+}\right)-X\left(f^{-}\right) \equiv \xi^{1}(\mathbf{a})-\xi^{2}(\mathbf{a}) .
$$

Given any element $\xi(a) \in A C$, we define

$$
f(\xi) \equiv Y[\xi(a)] \equiv Y\left[\xi^{+}(a)\right]-Y\left[\xi^{-}(a)\right] \equiv f^{1}(\xi)-f^{2}(\xi) .
$$

To prove that the transformations $X$ and $Y$ are inverse, we must show that $\xi^{1}(a)=\xi^{+}(a), \xi^{2}(a)=\xi^{-}(a), f^{1}(\xi)=f^{+}(\xi)$, and $f^{2}(\xi)=f^{-}(\xi)$; in other words, that

$$
\wedge\left[\xi^{1}(\boldsymbol{a}), \xi^{2}(\boldsymbol{a})\right]=\wedge\left[f^{1}(\xi), f^{2}(\xi)\right]=0
$$

Proof is omitted.

We have therefore a one-to-one correspondence between the elements of $A C$ and those of $\Lambda^{1}$, and $X^{-1}=Y, Y^{-1}=X$.

The transformation $\xi(a)=X(f)$ can now be represented in general in the form :

$$
\xi(a)=\int_{a} f d \mu
$$

THEOREM 5.10. The transformations $X$ and $Y$ preserve addition, ordering, and multiplication by real numbers. That is, $X$ and $Y$ are additive, homogeneous, and positive:

$$
X(\alpha f+\beta g)=\alpha X f+\beta X g, \quad Y(\alpha \xi+\beta \rho)=\alpha Y \xi+\beta Y \rho,
$$




$$
\begin{gathered}
f>0 \rightarrow X(f)>0, \\
\xi(a)>0 \rightarrow Y[\xi(a)]>0 .
\end{gathered}
$$

Proof. We need prove only: 1. $\int_{a}(\alpha f+\beta g) d \mu=\alpha \int_{a} f d \mu+\beta \int_{a} g d \mu$. 2. $f \geqq 0$ $\rightarrow \int_{a} f d \mu \geqq 0$, for every $a$. 3. $f>0 \rightarrow \int_{a} f d \mu>0$, for some $a .4$. $f$ not $\geqq 0 \rightarrow \int_{a} f d \mu<0$, for some $a$. Details of the proof are omitted.

This completes the proof that $A C$ is a space of type $F^{1}$, since it is isomorphic to $\Lambda^{1}$. Conversely, every space of type $F^{1}$ is isomorphic to a space of absolutely continuous functions.

Remark. If $a$ is an element of $B$, then the function of $A C$ which corresponds to the function $a$ of $\Lambda^{1}$ is the function

$$
\xi(b)=\mu[\wedge(a, b)] .
$$

\section{Concluding Remarks}

1. Regularity. Since a given Boolean algebra $B$ determines and is determined by the corresponding space $\Omega$, we may wish to know the conditions on $B$ corresponding to the regularity conditions on $\Omega$. A set of necessary and sufficient conditions is contained in the

Definition. A regular Boolean algebra is a $\sigma$-complete Boolean algebra $B$ which satisfies the further conditions:

1. If $F$ is any subset of $B$, then $\vee(F)$ exists, and there is a countable subset $F^{\prime} \subseteq F$ such that

$$
\bigvee\left(F^{\prime}\right)=\bigvee(F) .
$$

2. If $a_{n} \downarrow 0$ as $n \uparrow \infty, a_{n}^{k} \downarrow a_{n}$ as $k \uparrow \infty$, then there exists a subsequence, $a_{n}^{\boldsymbol{k}_{n}}$, such that

$$
\bigwedge_{n=1}^{\infty} \bigvee_{m=n}^{\infty} a_{m}^{k_{m}}=0
$$

3. If $a_{m}^{k}(n) \downarrow 0$ as $k \uparrow \infty$, for each $m, n$ and $a_{m}^{k}(n) \downarrow 0$ as $n \uparrow \infty$, for each $m, k$, then there exists a subsequence, $a_{m}^{k_{m}}(n)$, such that

$$
\bigwedge_{n=1}^{\infty} \bigvee_{m=1}^{\infty} a_{m}^{k_{m}}(n)=0
$$

Remark. A regular Boolean algebra is obviously complete.

THEOREM 6.1. A necessary and sufficient condition that $\Omega$ be regular is that $B$. be regular.

Proof is omitted.

We state, also without giving the proof:

Theorem 6.2. A Boolean algebra of type $B_{\mu}$ is regular. 
We know therefore that the space of measurable functions on the unit interval is regular, while the subspace of bounded functions is not.

2. Conjugate spaces. Riesz [10] has developed the theory of additive functionals defined on a "fundamental domain." Such a fundamental domain is given by the positive and zero elements of any vector lattice. Riesz proves, in different language, that the space of additive functionals, each bounded above by a positive additive functional, defined on a vector lattice, is a $\sigma$-complete vector lattice. If the underlying space is $\sigma$-complete we can restrict ourselves to linear (continuous) functionals. In this case each functional is automatically bounded above by a positive linear functional, and we can state

THEOREM 6.3. The space of linear functionals on a $\sigma$-complete vector lattice is a complete vector lattice.

Proof is omitted.

The notion of conjugate space becomes particularly interesting to us, because of the possibility of multiplying elements of $\Omega$, and because of the Nikodym theorem, in the case of the $\Lambda^{p}$ spaces. Following closely the methods given by Banach [11] for the $L^{p}$ spaces, we can prove the following theorems :

Theorem 6.4. (Hölder's inequality.) If $f \in \Lambda^{p}, g \in \Lambda^{q}, 1 / p+1 / q=1$, then $f g \in \Lambda^{1}$. If $f, g \geqq 0$, then

$$
\int f g d \mu \leqq\left[\int f^{p} d \mu\right]^{1 / p}\left[\int g^{q} d \mu\right]^{1 / q}
$$

Theorem 6.5. Every linear functional $A(f)$ on $\Lambda^{p}, p>1$, is of the form

$$
A(f)=\int f g d \mu
$$

where $g \in \Lambda^{q}, 1 / p+1 / q=1$, and the norm of $g$ in $\Lambda^{q}$ is

$$
\|g\|=\|A\| \text {. }
$$

THEOREM 6.6. Every linear functional $A(f)$ on $\Lambda^{1}$ is of the form

$$
A(f)=\int f g d \mu
$$

where $g \in M$, the space of bounded functions, and

$$
\|g\|=\|A\| \text {. }
$$

These theorems apply immediately to any spaces of type $F^{p}$. Many other theorems regarding $L^{p}$ spaces, such as those concerned with weak convergence (cf. Banach $\left[11\right.$, p. 197]) can be carried over to the $\Lambda^{p}$ spaces. Spaces of the type defined by Orlicz [4] can also be extended to spaces on a Boolean algebra of type $B_{\mu}$. 


\section{BIBLIOGRAPHY}

1. Hans Freudenthal, Teilweise geordnete Moduln, Proceedings, Akademie van Wetenschappen te Amsterdam, vol. 39 (1936), pp. 641-651.

2. S. Saks, Theory of the Integral, 1937.

3. L. V. Kantorovitch, Lineare halbgeordnete Räume, Recueil Mathématique, Moscow, (n.s.), vol. 2 (1937), pp. 121-165.

4. W. Orlicz, Über eine gewisse Klasse von Räumen vom Typus B, Bulletin International de l'Académie Polonaise des Sciences et des Lettres, A, 1932, pp. 207-220.

5. C. Carathéodory, Entwurf für eine Algebraisierung des Integralbegriffs, Sitzungsberichte der Bayerischen Akademie der Wissenschaften, 1938, pp. 27-69.

6. M. H. Stone, The theory of representations for Boolean algebras, these Transactions, vol. 40 (1936), pp. 37-111.

7. Franz Wecken, Abstrakte Integrale und fastperiodische Funktionen, Mathematische Zeitschrift, vol. 45.(1939), pp. 377-404.

8. Garrett Birkhoff, Lattice Theory, American Mathematical Society Colloquium Publications, vol. $25,1940$.

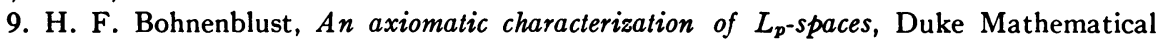
Journal, vol. 6 (1940), pp. 627-640.

10. Frédéric Riesz, Sur quelques notions fondamentales dans la théorie générale des opérations linéaires, Annals of Mathematics, (2), vol. 41 (1940), pp. 174-206.

11. S. Banach, Théorie des Opérations Linéaires, 1932.

UNIVERSITY OF MiNNESOTA, Minneapolis, Minn. 